\title{
介孔石墨相氮化碳载银聚醚砜膜制备及性能研究
}

\author{
刘姿铔，曹如雅，张曼莹 \\ (江苏理工学院化学与环境工程学院, 常州 213001)
}

摘 要: 膜污染一直是膜分离应用中的主要问题。将不同量的介孔石墨相氮化碳载银 $\left(\mathrm{m}-\mathrm{g}-\mathrm{C}_{3} \mathrm{~N}_{4} / \mathrm{Ag}\right)$ 以共混法引入 铸膜液中, 通过相转化法制备聚梄砜(PES)纳米复合膜, 系统研究了 $\mathrm{m}-\mathrm{g}-\mathrm{C}_{3} \mathrm{~N}_{4} / \mathrm{Ag}$ 的添加对纳米复合膜形貌、过滤、 抗菌、光催化和抗污染性能的影响。结果表明, $\mathrm{m}-\mathrm{g}-\mathrm{C}_{3} \mathrm{~N}_{4} / \mathrm{Ag}$ 的添加可以改善纳米复合膜的断面结构及表面亲水性。 与纯 PES 膜相比, 纳米复合膜纯水通量随着掺杂量的增加显著提高, 各个样品对蛋白质的截留率均在 $90 \%$ 以上, 表明 $\mathrm{m}-\mathrm{g}-\mathrm{C}_{3} \mathrm{~N}_{4} / \mathrm{Ag}$ 的添加在不影响截留性能的前提下，可以显著提高纳米复合膜的过滤性能。纳米复合膜的抗菌 性能随着 $\mathrm{m}-\mathrm{g}-\mathrm{C}_{3} \mathrm{~N}_{4} / \mathrm{Ag}$ 含量的增加而提高, 其中对铜绿假单胞菌的抗菌效果明显高于大肠杆菌。纯 PES 膜在光照 下几乎不发生光降解。相比之下, 所有添加 $\mathrm{m}-\mathrm{g}-\mathrm{C}_{3} \mathrm{~N}_{4} / \mathrm{Ag}$ 的纳米复合膜在可见光照射下均呈现良好的光催化性能, 且光催化活性随着 $\mathrm{m}-\mathrm{g}-\mathrm{C}_{3} \mathrm{~N}_{4} / \mathrm{Ag}$ 的增加而逐渐增强。其中 $\mathrm{m}-\mathrm{g}-\mathrm{C}_{3} \mathrm{~N}_{4} / \mathrm{Ag}$ 添加量最高的纳米复合膜显示出最明显的 光催化作用，在 $120 \mathrm{~min}$ 内甲基橙的脱色率可达 $63 \%$ 。通过四步过滤实验对所有膜的综合抗污染性能进行表征，可 知所有纳米复合膜通量恢复率均显著高于纯 PES 膜。水洗和可见光照射后所有膜的膜通量恢复率皆进一步提高。 综上所述, 添加 $\mathrm{m}-\mathrm{g}-\mathrm{C}_{3} \mathrm{~N}_{4} / \mathrm{Ag}$ 可以显著提高聚醚砜膜的抗菌性、可见光下光催化降解染料性能, 进而改善其综合 抗污染性能。

关 键 词: 介孔石墨相氮化碳载银; 聚醚砜膜; 抗菌; 光催化; 抗污染

中图分类号: TQ028 文献标识码: A

\section{Preparation and Property of Polyethersulfone Ultrafiltration Membranes with Mesoporous-graphitic- $\mathrm{C}_{3} \mathrm{~N}_{4} / \mathrm{Ag}$}

\author{
LIU Zi-Ya, CAO Ru-Ya, ZHANG Man-Ying
}

(School of Chemical and Environmental Engineering, Jiangsu University of Technology, Changzhou 213001, China)

\begin{abstract}
Membrane fouling is still the major problem for the application of membrane separation. In this study, different amounts of silver doped mesoporous graphitic carbon nitride (m-g- $\left.\mathrm{C}_{3} \mathrm{~N}_{4} / \mathrm{Ag}\right)$ were introduced into casting solution by blending. The polyethersulfone (PES) nanocomposite membrane was prepared via the phase-inversion method. The impacts of m-g- $\mathrm{C}_{3} \mathrm{~N}_{4} / \mathrm{Ag}$ addition on the morphology, filtration, antibacterial, photocatalytic, and antifouling properties of nanocomposite membrane were systematically studied. The results showed that the addition of m-g- $\mathrm{C}_{3} \mathrm{~N}_{4} / \mathrm{Ag}$ can improve the cross-section structure of the nanocomposite membrane as well as its surface
\end{abstract}

收稿日期：2018-07-06; 收到修改稿日期：2018-10-25

基金项目：国家自然科学基金青年基金(51508239); 江苏省自然科学基金青年基金(BK20150245); 江苏省研究生教育教学 改革课题(JGLX18-165、JGLX18-166); 江苏省研究生科研与实践创新计划项目(SJCX17-0772); 江苏省“青蓝工 程”项目

National Natural Science Foundation of China (51508239); Natural Science Foundation of Jiangsu Province (BK20150245); Postgraduate Education Reform Project of Jiangsu Province(JGLX18-165, JGLX18-166); Postgraduate Research \& Practice Innovation Program of Jiangsu Province (SJCX17-0772); Qing Lan Project

作者简介：刘姿铔(1994-)，女，硕士研究生. E-mail: 270259089@qq.com

通讯作者：张曼䒯，讲师. E-mail: myzhang@jsut.edu.cn 
hydrophilicity. Compared with pure PES membrane, the pure water flux of the modified nanocomposite membrane increased significantly with the increase of doping amount. The protein rejection rates of all samples were over $90 \%$, indicating that the addition of $\mathrm{m}-\mathrm{g}-\mathrm{C}_{3} \mathrm{~N}_{4} / \mathrm{Ag}$ can significantly improve the filtration performance of the nanocomposite membrane without sacrificing the rejection performance. The anti-bacterial activity of the nanocomposite membranes was improved with the increase of m-g- $\mathrm{C}_{3} \mathrm{~N}_{4} / \mathrm{Ag}$ content in which the anti-bacterial activity to $P$. aeruginosa was much more significant than that to E. coli. The pure PES membrane almost had not photodegradation under light irradiation. By contrast, all nanocomposite membranes exhibited good photocatalytic properties under visible light irradiation, and the photocatalytic activity improved with increase of $\mathrm{m}-\mathrm{g}-\mathrm{C}_{3} \mathrm{~N}_{4} / \mathrm{Ag}$. The nanocomposite membrane in which the m-g- $\mathrm{C}_{3} \mathrm{~N}_{4} / \mathrm{Ag}$ content was the highest that showed the optimal photocatalysis, and the decolorization rate of methyl orange reached $63 \%$ in $120 \mathrm{~min}$. The comprehensive antifouling performances of all membranes were characterized by four-step filtration experiments. It can be seen that the flux recovery ratio $(F R R)$ of all nanocomposite membranes is much higher than that of PES membrane. The FRR of all membranes was further increased after washing with water and irradiating with visible light. In summary, the addition of m-g- $\mathrm{C}_{3} \mathrm{~N}_{4} / \mathrm{Ag}$ can endow the PES membrane with the antibacterial and the photocatalytic properties under visible light irradiation, thereby improving its comprehensive antifouling performance.

Key words: $\mathrm{m}-\mathrm{g}-\mathrm{C}_{3} \mathrm{~N}_{4} / \mathrm{Ag}$; polyethersulfone membrane; antibiosis; photocatalysis; antifouling

膜分离技术以其操作简单、高效节能等优点在 饮用水及污水处理领域得到了广泛应用 ${ }^{[1-3]}$ 。聚醚砜 (PES)具有良好的化学稳定性、耐热性、抗老化性和 较好的加工性，被广泛用于膜材料的制备 ${ }^{[4]}$ 。但是同 其他常见的膜材料一样, 聚醚砜膜也面临着“膜污 染”的危害。膜污染会严重影响膜组件的过滤截留性 能, 此外工业上为了减轻膜污染, 保证分离过程顺 利进行, 往往需要提高跨膜压(TMP), 并对污染后 的膜组件进行物理反冲洗或化学清洗, 这会增加能 耗及运行成本 ${ }^{[5]}$ 。因此, 提高膜材料的抗污染性能得 到了越来越多的关注。

近年来, 随着纳米技术的发展, 纳米掺杂耐污 染膜材料作为一种源头控制膜污染的方法成为研究 的热点 ${ }^{[6]}$ 。纳米二氧化硅 ${ }^{[7]} 、 \mathrm{TiO}_{2}{ }^{[8-9]}$ 、石墨烯 ${ }^{[10]}$ 、 纳米银 ${ }^{[11]}$ 等具有较好的亲水性、化学稳定性、光催 化效应、抗菌性等而均被成功引入到膜材料中。研 究表明, 添加纳米材料不仅可以改变膜材料的表面 特性和断面孔结构, 提高过滤性能, 而且可以整合 纳米材料的特殊性能, 使膜不再是一个简单的物理 屏障, 还具有抗菌性和光催化等特殊性能。

石墨相氮化碳 $\left(\mathrm{g}-\mathrm{C}_{3} \mathrm{~N}_{4}\right)$ 因其优异的化学稳定性、 热稳定性、可见光下光催化性能得到了广泛关注。 但是单一组分的石墨相氮化碳通常面临着太阳能利 用率较低, 光生电子-空穴复合率高, 量子效率低 等问题, 从而削弱了其光催化活性。因此, 通过对光 催化剂进行改性, 拓展其光吸收范围、抑制光生载
流子复合, 已成为材料、化学与环境等学科的研究 热点 ${ }^{[12]}$ 。而以 $\mathrm{g}-\mathrm{C}_{3} \mathrm{~N}_{4}$ 为载体, 用金属 $\left(\mathrm{Ag}^{[13]} 、 \mathrm{Pt}^{[14]}\right.$ 、 $\mathrm{Pd}^{[15]}$ 等)对其表面进行修饰, 可以有效地抑制光生 电子-空穴对的复合，提高其光催化活性。此外，介 孔石墨相氮化碳 $\left(\mathrm{m}-\mathrm{g}-\mathrm{C}_{3} \mathrm{~N}_{4}\right)$ 依靠可进入的多孔性和 较高的比表面积提供更多的反应活性位点使其光催 化性能得到提高 ${ }^{[16]}$; 而将此类新型纳米复合物作为 添加剂制备纳米复合膜材料的研究尚不多见。

纳米银具有广谱杀菌性, 超强的活性和渗透性, 在抗污染膜材料中得到充分的应用。纳米银已经被 成功引入到聚醚砜 $(\mathrm{PES})^{[17]}$ 、聚酰胺 $(\mathrm{PA})^{[18]}$ 、醋酸纤 维素 $(\mathrm{CA})^{[19]}$ 等多种聚合物膜材料中。研究结果表 明, 添加纳米银可以有效提高复合膜的抗菌及抗 生物污染性能。目前, 研究者认为纳米银掺杂复合 膜的抗菌性主要源于两方面：一是细菌与纳米银 的直接接触; 二是复合膜中纳米银向周围介质释放 对微生物有抑制作用的 $\mathrm{Ag}^{+}$, 而 $\mathrm{Ag}^{+}$能够和蛋白质 中颈基(-SH)结合, 导致呼吸酶失活, 阻断 DNA 的 复制等 ${ }^{[20-21]}$ 。

基于此, 本研究以膜污染控制为出发点, 通过 介孔石墨相氮化碳 $\left(\mathrm{m}-\mathrm{g}-\mathrm{C}_{3} \mathrm{~N}_{4}\right)$ 载银纳米复合材料的 构建, 将 $\mathrm{m}-\mathrm{g}-\mathrm{C}_{3} \mathrm{~N}_{4}$ 的光催化性能与纳米银的杀菌性 有机结合, 开展新型的高效光催化抗污染纳米复合 膜材料的制备及性能研究，探索剖析纳米复合材料 添加量对复合膜材料结构、过滤以及综合抗污染性 能的影响。 


\section{1 实验方法}

\section{1 仪器与药品}

仪器: 纯水仪 (Basic-Q15-IT, 上海和泰仪器有 限公司); 小型高速离心机(LX-300, Kylin-Bell Lab Instruments CO, LTD. ); 可见分光光度计(D-7, 南 京菲勒仪器有限公司); 超净工作台 (SW-CJ-1FD, 苏州安泰空气技术有限公司); 恒温培养摇床 (THZ-103B, 上海一恒科学仪器有限公司); 电热恒 温培养箱(DHP-9032, 上海一恒科学仪器有限公司); 立式压力蒸汽灭菌器(LDZF-50KB, 上海申安医疗 器械厂); 超滤杯 (8010, 德国默克集团)。

药品: 介孔石墨相氮化碳(自制)、介孔石墨相氮 化碳载银(自制)、甲基橙、氯化钠、磷酸氢二钾、 磷酸二氢钾、硫酸铵、柠檬酸钠、七水硫酸镁、二 甲基甲酰胺均为分析纯, 购自国药集团化学试剂有 限公司。蛋白炼、酵母提取物、Agar 购自英国 OXOID 公司。银标准溶液 $\left(1000 \mathrm{mg} \cdot \mathrm{L}^{-1}\right)$ 购自国家有色金属 及电子材料分析测试中心。PES 购自德国巴斯夫。 本实验以大肠杆菌(Escherichia coil ATCC 15597)和 铜绿假单胞菌(Pseudomonas aeruginosa ATCC 27853) 为测试菌。

\section{$1.2 \mathrm{~m}-\mathrm{g}-\mathrm{C}_{3} \mathrm{~N}_{4} / \mathrm{Ag}$ 制备}

将 $5 \mathrm{~g}$ 三聚氰胺和 $5 \mathrm{~g} \mathrm{NH}_{4} \mathrm{Cl}$ 在玛瑙研钵中研磨, 使其混合均匀。然后, 将所得均匀固体放入有盖瓷 碗中, 在 $600{ }^{\circ} \mathrm{C}$ 马弗炉中加热 $2 \mathrm{~h}$, 得到介孔石墨相 氮化碳 ${ }^{[22]}$ 。

首先, 取 $0.3 \mathrm{~g}$ 制备的 $\mathrm{m}-\mathrm{g}-\mathrm{C}_{3} \mathrm{~N}_{4}$ 加入 $100 \mathrm{~mL}$ 去 离子水中, 超声 $30 \mathrm{~min}$, 使其均匀分散。然后, 将 $15 \mathrm{~mL} \mathrm{AgNO}_{3}$ 水溶液和 $10 \mathrm{~mL}$ 无水甲醇加至 $\mathrm{m}-\mathrm{g}-$ $\mathrm{C}_{3} \mathrm{~N}_{4}$ 液中, 得到的混合液在高压录灯照射下搅拌 $12 \mathrm{~h}$ 后离心并用去离子水洗涤三次, 进行纯化。最后, 将产物置于 $70{ }^{\circ} \mathrm{C}$ 的真空干燥箱中干燥 $12 \mathrm{~h}$ 备用 ${ }^{[23]}$ 。

\section{$1.3 \mathrm{~m}-\mathrm{g}-\mathrm{C}_{3} \mathrm{~N}_{4} / \mathrm{Ag} / \mathrm{PES}$ 膜制备}

所有膜通过湿法相转化法制备。将不同质量的 $\mathrm{m}-\mathrm{g}-\mathrm{C}_{3} \mathrm{~N}_{4} / \mathrm{Ag}$ 粉末加入到 $N, N$-二甲基甲酰胺(DMF) 中并通过摚拌充分分散。然后在混合液中加入相同 质量的 PES, 置于 $60{ }^{\circ} \mathrm{C}$ 烘箱中加热直至完全溶解。 将溶解的铸膜液搅拌至透明状后在 $60{ }^{\circ} \mathrm{C}$ 下静置脱 泡。待铸膜液冷却至室温后, 用刮刀均匀地刮在无 纺布上, 然后将其迅速浸入纯水中固化成膜并浸泡 $24 \mathrm{~h}$, 以使溶剂交换和相转化完全 ${ }^{[24]}$ 。表 1 为本实 验中铸膜液组成成分。
表 1 实验中铸膜液组成

Table 1 Composition of dope solution

\begin{tabular}{cccc}
\hline $\begin{array}{c}\text { Membrane } \\
\text { abbreviation }\end{array}$ & $\begin{array}{c}\text { Silver } \\
\text { content/wt\% }\end{array}$ & PES/wt\% & DMF/wt\% \\
\hline M0 & 0 & 18 & 82.0 \\
M1 & 0.1 & 18 & 81.9 \\
M2 & 0.3 & 18 & 81.7 \\
M3 & 0.5 & 18 & 81.5 \\
M4 & 1.0 & 18 & 81.0 \\
\hline
\end{tabular}

\section{$1.4 \mathrm{~m}-\mathrm{g}-\mathrm{C}_{3} \mathrm{~N}_{4} / \mathrm{Ag} / \mathrm{PES}$ 膜表征}

采用扫描电子显微镜(SEM, HITACHI S-4800) 观察不同含量 $\mathrm{m}-\mathrm{g}-\mathrm{C}_{3} \mathrm{~N}_{4} / \mathrm{Ag}$ 对膜表面及断面形貌的 影响，通过接触角测量仪(CAM200, KSV)测试膜表 面的亲疏水性, 每个样品至少选取 6 个测试点以减 小实验误差。

孔隙率定义为膜孔体积占膜总体积的百分数, 公式如(1)所示:

$$
\varepsilon=\frac{\left(W_{\mathrm{w}}-W_{\mathrm{d}}\right)}{A L d_{\mathrm{w}}} \times 100 \%
$$

式中, $\varepsilon$ 为孔隙率, $\% ; W_{\mathrm{w}}$ 为湿膜重量, $\mathrm{g} ; W_{\mathrm{d}}$ 为干膜 重量, $\mathrm{g} ; A$ 为膜面积, $\mathrm{m}^{2} ; L$ 为膜的厚度, $\mathrm{m} ; d_{\mathrm{w}}$ 为室温 下纯水密度, $\mathrm{g} \cdot \mathrm{cm}^{-3}$ 。

吸水率 $U(\%)$ 是用来评价多孔材料吸水性能的 指标, 公式如(2)所示:

$$
U=\left(\frac{W_{\mathrm{w}}-W_{\mathrm{d}}}{W_{\mathrm{w}}}\right) \times 100 \%
$$

式中, $U$ 为吸水率, $\% ; W_{\mathrm{w}}$ 为湿膜重量, $\mathrm{g} ; W_{\mathrm{d}}$ 为干膜 重量, g。

截留分子量(MWCO)是表征超滤膜孔径大小的 重要指标，通过测量不同大小蛋白质的截留率，将 截留率在 $90 \%$ 以上的最小分子量定义为膜的截留分 子量, 公式如(3)所示:

$$
R=\left(1-\frac{C_{\mathrm{P}}}{C_{\mathrm{f}}}\right) \times 100 \%
$$

式中, $C_{\mathrm{p}}$ 为透过液蛋白质浓度, $\mathrm{g} / \mathrm{L} ; C_{\mathrm{f}}$ 为原液蛋白质 浓度, $\mathrm{g} / \mathrm{L}$ 。实验采用卵清蛋白(45 kDa), 牛血清蛋白 $(69 \mathrm{kDa})$ 进行测试, 所有蛋白质溶液 $(1 \mathrm{~g} / \mathrm{L})$ 用磷酸 盐缓冲溶液配置。

采用死端过滤装置进行纯水过滤, 实验条件如 下: 将膜在 $0.2 \mathrm{MPa}$ 下纯水预压 $0.5 \mathrm{~h}$, 然后在 $0.1 \mathrm{MPa}$ 、滤杯中转子转速始终保持在 $400 \mathrm{r} / \mathrm{min}$ 下 测其纯水通量 $(J)$, 公式如(4)所示:

$$
J=\frac{V}{A \Delta t}
$$

式中, $J$ 为膜通量, $\mathrm{L} \cdot \mathrm{m}^{-2} \cdot \mathrm{h}^{-1} ; V$ 为 $\Delta t$ 内透过液体的体 
积, $\mathrm{L} ; A$ 为膜的有效过滤面积, $\mathrm{m}^{2} ; \Delta t$ 为过滤时间, $\mathrm{h}$ 。

\section{5 动态银释放测试}

纳米银由于亲水性易向水相介质迁移, 在过滤 过程中, 由于操作压力的存在, 膜中一些纳米银或 释放的 $\mathrm{Ag}^{+}$会随滤液迁出, 造成纳米银的损失。为 了评价复合膜在过滤过程中的银释放行为, 本实验 用死端过滤装置进行纯水过滤 ${ }^{[25]}$ 。

\section{6 抗菌性能测试}

抑菌环法是用于表征复合膜抗菌性能的最直观 的方法 ${ }^{[26]}$ 。实验中以革兰氏阴性菌大肠杆菌 (E.coil) 和铜绿假单胞菌 (P. aeruginosa) 为测试菌。将菌种在 Luria-Bertani (LB)培养液中置于 $37{ }^{\circ} \mathrm{C}$ 摇床中过夜培 养, 吸取 $100 \mu \mathrm{L}$ 菌液用涂布棒均匀地涂布在 $\mathrm{LB}$ 固 体平板上, 然后将面积为 $4.1 \mathrm{~cm}^{2}$ 的膜片正面朝下平 放在涂有菌液的平板上, 在 $37{ }^{\circ} \mathrm{C}$ 培养箱中培养 $24 \mathrm{~h}$ 。注意, 所有膜片样品在使用前经紫外光照射杀 菌 $30 \mathrm{~min} 。 24 \mathrm{~h}$ 后, 拍照记录膜片周围形成的抑菌 环, 每个样品重复三次。

CFU 计数法可以更精确地表征复合膜抗菌性 能。实验开始前, 所有膜片样品在紫外光下照射 $30 \mathrm{~min}$ 。将过夜培养的菌液 $1 \mathrm{~mL}$ 加入 $100 \mathrm{~mL}$ Minimal Davis (MD)介质中, 然后将 2 片面积为 $4.1 \mathrm{~cm}^{2}$ 的膜片放入其中, 并放置于 $37{ }^{\circ} \mathrm{C}$ 摇床中培养 $12 \mathrm{~h}$ 。 培养结束后, 将混合液用 $\mathrm{MD}$ 溶液进行梯度稀释至 合适浓度, 取 $100 \mu \mathrm{L}$ 稀释后的液体用涂布棒均匀涂 布在 LB 固体平板上, $37{ }^{\circ} \mathrm{C}$ 培养箱中培养 $24 \mathrm{~h}$ 后对 平板上菌落数进行计数 ${ }^{[27]}$ 。每个样品重复三次, 求 其平均值。抑菌率(B)计算公式如(5)所示:

$$
B=\left(1-\frac{N_{\mathrm{n}}}{N_{\mathrm{p}}}\right) \times 100 \%
$$

式中, $B$ 为抑菌率, $\% ; N_{\mathrm{n}}$ 为 $\mathrm{m}-\mathrm{g}-\mathrm{C}_{3} \mathrm{~N}_{4} / \mathrm{Ag} / \mathrm{PES}$ 膜的菌落 数, $\mathrm{CFU} \mathrm{mL}{ }^{-1} ; N_{\mathrm{p}}$ 为纯 $\mathrm{PES}$ 膜的菌落数, $\mathrm{CFU} \mathrm{mL}{ }^{-1}$ 。

\section{7 光催化测试}

通过测定甲基橙(MO) 在可见光照射下的降解 效率来评价各种膜的光催化性能。首先, 使用双面 胶将膜 $\left(15 \mathrm{~cm}^{2}\right)$ 固定在载玻片上, 并将其与 $100 \mathrm{~mL}$ 的 $\mathrm{MO}$ 溶液 $(10 \mathrm{mg} / \mathrm{L})$ 一同置于光催化反应器中, 在 室温下黑暗静置 $30 \mathrm{~min}$, 以保证达到吸附一脱附平 衡。然后用 $300 \mathrm{~W}$ 氙灯(用 $420 \mathrm{~nm}$ 截止滤光片得到 可见光)照射, 膜样品与光源保持 $15 \mathrm{~cm}$ 的垂直距离, 适当的时间间隔取样 $3 \mathrm{~mL}$, 通过 UV-Vis 对所取清 液在 $464 \mathrm{~nm}$ 处测定其吸光度, 并通过染料的标准曲 线方程, 计算得出不同光照时间下的染料浓度及其 光催化脱色率 ${ }^{[25]}$ 。

\section{8 抗污染性能测试}

为比较各种膜的抗污染性能, 进行如下实验: 首先, 测量纯水通量直至通量 $\left(J_{\mathrm{w} 1}\right)$ 稳定; 然后, 将 BSA 溶液 $(1 \mathrm{~g} / \mathrm{L})$ 加入过滤系统, 测量 $1 \mathrm{~h}$ 通量 $\left(J_{\mathrm{p}}\right)$; 再用去离子水漂洗污垢膜以除去松散蛋白质后测纯 水通量 $\left(J_{\mathrm{rw}}\right)$ 。最后, 为了证明纳米复合膜的光催化 抗污性能, 将 BSA 污染膜用可见光照射 $0.5 \mathrm{~h}$ 后, 再 测其纯水通量 $\left(J_{\mathrm{w} 2}\right)$ 。采用流量回收率 $(F R R)$ 、总污染 率 $\left(R_{\mathrm{t}}\right)$ 、可逆结垢率 $\left(R_{\mathrm{r}}\right)$ 、不可逆结垢率 $\left(R_{\mathrm{ir}}\right)$ 分析纳 米复合膜的抗污染性能, 具体公式如下 ${ }^{[26]}$.

$$
\begin{gathered}
F R R=\left(\frac{J_{\mathrm{w} 2}}{J_{\mathrm{w} 1}}\right) \times 100 \% \\
R_{\mathrm{t}}=\left(1-\frac{J_{\mathrm{P}}}{J_{\mathrm{w} 1}}\right) \times 100 \%=R_{\mathrm{r}}+R_{\mathrm{ir}} \\
R_{\mathrm{r}}=\left(\frac{J_{\mathrm{w} 2}-J_{\mathrm{p}}}{J_{\mathrm{w} 1}}\right) \times 100 \% \\
R_{\mathrm{ir}}=\left(\frac{J_{\mathrm{w} 1}-J_{\mathrm{w} 2}}{J_{\mathrm{w} 1}}\right) \times 100 \%
\end{gathered}
$$

\section{2 结果与讨论}

\section{1 纳米复合膜表征}

\subsection{1 纳米复合膜形貌表征}

如图 1(a)所示, 纯 PES 膜(M0)具有光滑且干净 的表面。随着 $\mathrm{m}-\mathrm{g}-\mathrm{C}_{3} \mathrm{~N}_{4} / \mathrm{Ag}$ 含量的增加, 纳米复合 膜表面出现一些白色颗粒，这可能是 $\mathrm{m}-\mathrm{g}-\mathrm{C}_{3} \mathrm{~N}_{4} / \mathrm{Ag}$ 纳米片的聚集体, 但纳米复合膜的表面形貌整体上 仍然平整光滑, 表明添加 $\mathrm{m}-\mathrm{g}-\mathrm{C}_{3} \mathrm{~N}_{4} / \mathrm{Ag}$ 对表面结构 影响不大。

从图 1(b)中可见, 添加 m-g- $\mathrm{C}_{3} \mathrm{~N}_{4} / \mathrm{Ag}$ 会对复合 膜断面结构产生略微影响。所有样品均呈现典型的 非对称结构。表层为致密选择层, 中间为指状多孔 亚层, 底部为海绵层。此外, 加入 $\mathrm{m}-\mathrm{g}-\mathrm{C}_{3} \mathrm{~N}_{4} / \mathrm{Ag}$ 后复 合膜底部海绵层厚度减小, 且指状孔结构的孔径增 大、孔道加长。这是由于在相转化过程中, m-g$\mathrm{C}_{3} \mathrm{~N}_{4} / \mathrm{Ag}$ 加快了 $\mathrm{DMF}$ 与纯水之间的传质速度, 进而 有助于形成大孔结构 ${ }^{[28]}$ 。膜厚度为 $0.13 \sim 0.15 \mathrm{~mm}$ 。

\subsection{2 m-g- $\mathrm{C}_{3} \mathrm{~N}_{4} / \mathrm{Ag}$ 含量对纳米复合膜性能的影响}

表面亲水性是决定膜通量和抗污性能的重要因 素。通常采用接触角来评估膜表面的亲水性, 较小 的接触角意味着较高的亲水性。表 2 比较了各种膜 的接触角, $\mathrm{m}-\mathrm{g}-\mathrm{C}_{3} \mathrm{~N}_{4} / \mathrm{Ag} / \mathrm{PES}$ 膜的接触角均小于 PES 膜的接触角。M0 的接触角为 $68.5^{\circ}$, 这与文献[27] 的结果一致。随着 $\mathrm{m}-\mathrm{g}-\mathrm{C}_{3} \mathrm{~N}_{4} / \mathrm{Ag}$ 的加入, 接触角 
(a)
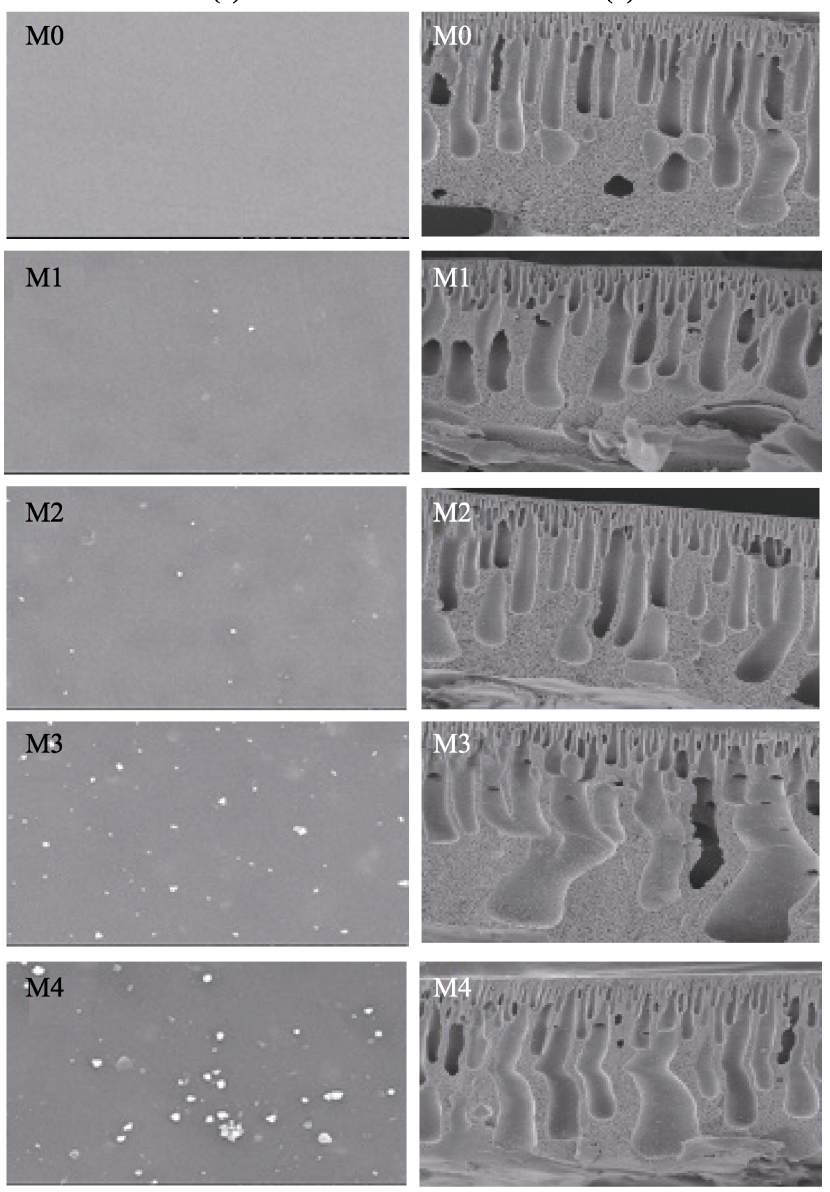

图 1 复合膜的表面(a)和断面形貌(b)

Fig. 1 Morphologies of composite membranes (a) Surface; (b) Cross section

逐渐减小, 表明 $\mathrm{m}-\mathrm{g}-\mathrm{C}_{3} \mathrm{~N}_{4} / \mathrm{Ag}$ 提高了膜的亲水性。 $\mathrm{m}-\mathrm{g}-\mathrm{C}_{3} \mathrm{~N}_{4} / \mathrm{Ag}$ 中亲水基团如 $-\mathrm{NH}_{2}$ 或- $\mathrm{NH}$ 的存在，可 能是亲水性增加的原因 ${ }^{[29]}$ 。

表 2 还列出了各种膜的孔隙率, m-g- $\mathrm{C}_{3} \mathrm{~N}_{4} / \mathrm{Ag} / \mathrm{PES}$ 膜的孔隙率均大于 PES 膜的孔隙率, 这是由于在成 膜过程中, 亲水性 $\mathrm{m}-\mathrm{g}-\mathrm{C}_{3} \mathrm{~N}_{4} / \mathrm{Ag}$ 的加入加快了非溶 剂和溶剂之间的交换速率, 使水向膜内传递的速度 加快, 不仅会形成大的孔道, 而且使得膜的孔隙率 增加, 这与横截面孔隙结构变化一致。

此外, 由表 2 可知, 复合膜表面吸水率相比纯

表 2 纳米复合膜的基本性能

Table 2 Basic specifications of the membranes

\begin{tabular}{ccccc}
\hline $\begin{array}{c}\text { Membrane } \\
\text { abbreviation }\end{array}$ & $\mathrm{CA} /\left(^{\circ}\right)$ & $\begin{array}{c}\text { Porosity } \\
/ \%\end{array}$ & $\begin{array}{c}\text { Water } \\
\text { uptake } / \%\end{array}$ & $\begin{array}{c}\text { MWCO } \\
/ \mathrm{kDa}\end{array}$ \\
\hline M0 & $68.50 \pm 1.64$ & 63.31 & 31.77 & 69 \\
M1 & $63.03 \pm 1.70$ & 64.06 & 32.52 & 69 \\
M2 & $60.27 \pm 1.90$ & 64.51 & 32.94 & 69 \\
M3 & $55.66 \pm 2.60$ & 65.38 & 33.79 & 69 \\
M4 & $53.75 \pm 4.70$ & 66.04 & 34.42 & 69 \\
\hline
\end{tabular}

PES 膜有所增加。复合膜表面亲水性和孔隙率的增 加必然会改善膜表面吸水能力。

采用死端过滤装置来研究各种膜样品的过滤性 能, 结果如图 2 所示。与 $\mathrm{M} 0$ 相比, 纳米复合膜的通 量保持持续增加，增幅皆大于 $30 \%$, 并且 M4 具有 最高通量 $258.29 \mathrm{~L} \cdot \mathrm{m}^{-2} \cdot \mathrm{h}^{-1}$ 。基于所有膜的 $\mathrm{MWCO}$ 皆为 $69 \mathrm{kDa}$, 因此膜样品的通量变化是由于 m-g$\mathrm{C}_{3} \mathrm{~N}_{4} / \mathrm{Ag}$ 的引入提高了纳米复合膜的亲水性和孔隙 率，并且扩大了指状孔隙，改善了断面孔隙结构的 贯通性，降低了水力阻力，增加了水通量。由图 2 可看出, 各种膜的 BSA 截留率皆大于 $90 \%$, 表明 $\mathrm{m}-\mathrm{g}-\mathrm{C}_{3} \mathrm{~N}_{4} / \mathrm{Ag}$ 的添加是在不牺牲 BSA 截留率的情况 下提高水通量的有效途径。

\section{2 动态银释放}

图 3 为过滤过程中银释放数据, 可见银离子逐 渐从复合膜表面释放, 在最初的 $8 \mathrm{~h}$, 释放速率相对 较高，随着越来越多的水被过滤，滤液中银离子的 浓度逐渐降低。对于所有膜样品，银离子的释放量 与 $\mathrm{m}-\mathrm{g}-\mathrm{C}_{3} \mathrm{~N}_{4} / \mathrm{Ag}$ 添加量成正比。根据中华人民共和 国自来水水质国家标准(GB5749-2006)规定，饮用 水中银含量不得超过 $50 \times 10^{-9}$ 。而经过 $3 \mathrm{~h}$ 的过滤, 所有膜样品中释放的银含量都在 $50 \times 10^{-9}$ 以下，所

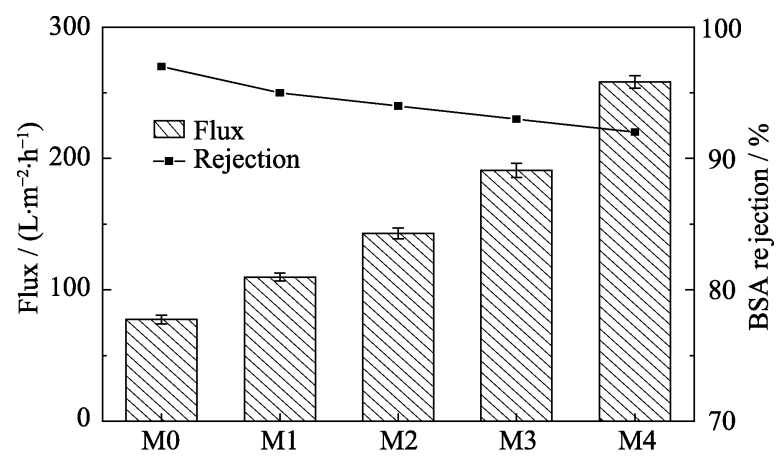

图 2 膜的纯水通量和 BSA 截留率

Fig. 2 Pure water flux and BSA rejection of membranes

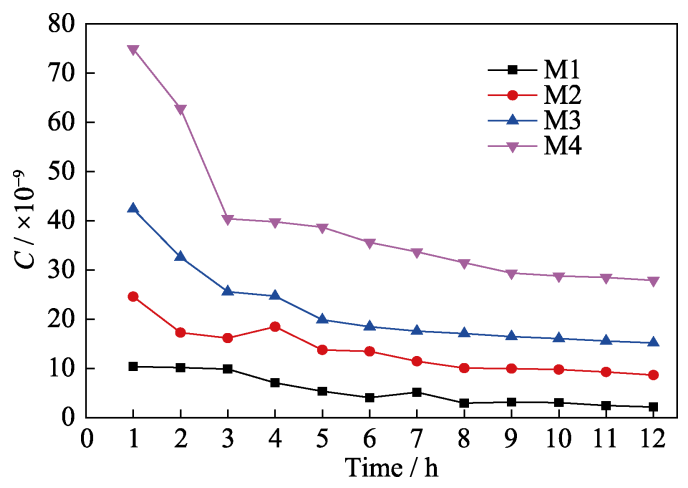

图 3 各个膜样品在 $12 \mathrm{~h}$ 过滤过程中银释放的浓度变化曲线 Fig. 3 Concentrations of leaching silver during $12 \mathrm{~h}$ filtration of each membrane sample 
以 $\mathrm{m}-\mathrm{g}-\mathrm{C}_{3} \mathrm{~N}_{4} / \mathrm{Ag} / \mathrm{PES}$ 复合膜不会造成二次污染，且 可以用于饮用水中膜生物污染的控制。一般认为, 过滤过程中银的损失主要来自于膜表面, 而释放的 $\mathrm{Ag}^{+}$是主要的杀菌剂 ${ }^{[30]}$ 。从膜表面 SEM 照片可知, 随着铸膜液中 $\mathrm{m}-\mathrm{g}-\mathrm{C}_{3} \mathrm{~N}_{4} / \mathrm{Ag}$ 含量增加, 膜表面银浓 度也明显增加, 更多的 $\mathrm{Ag}^{+}$得以释放。这为后期研 究复合膜的抗菌性能提供了研究基础。

\section{3 抗菌性能}

\subsection{1 抑菌环法}

本实验选用 P.aeruginosa 和 E.coli 为测试菌, 结 果如图 4 所示。纯 PES 膜周围无抑菌环生成, 表明 纯 PES 膜对铜绿假单胞菌和大肠杆菌的生长无任何 抑制作用。所有纳米复合膜都具有清晰的抑菌环, 且抑菌环宽度与 $\mathrm{m}-\mathrm{g}-\mathrm{C}_{3} \mathrm{~N}_{4} / \mathrm{Ag}$ 含量成正比, 表明复 合膜对大肠杆菌和铜绿假单胞菌均具有显著的抑制 能力, 且复合膜的抗菌活性与 $\mathrm{m}-\mathrm{g}-\mathrm{C}_{3} \mathrm{~N}_{4} / \mathrm{Ag}$ 添加量 呈正相关性 ${ }^{[28]}$ 。此外, (b)组的抑菌环明显大于(a)组, 说明复合膜对 P.aeruginosa 具有更强的杀菌能力。

\subsubsection{CFU 计数法}

表 3 给出了纳米复合膜的抗菌性能, 如表 3 所
示，复合膜都具有较高的抑菌率，且随着 $\mathrm{m}-\mathrm{g}$ $\mathrm{C}_{3} \mathrm{~N}_{4} / \mathrm{Ag}$ 含量的增加, 抑制细菌生长效应更为显著, 特别是 M3 和 M4, 其抑菌率高达 $100 \%$ 。此外, 复 合膜对铜绿假单胞菌的抑菌率皆明显高于大肠杆菌, 表明复合膜对铜绿假单胞菌具有更好的杀菌效果。

上述结果表明, 复合膜具有优异的抗菌性能。 将纳米银作为抗菌剂与膜材料结合的报道有很多, 但研究者对纳米银在膜材料中的杀菌机制并没有统 一的认识。有研究者认为纳米复合膜中的纳米银能 从膜表面扩散到周围介质中 ${ }^{[23]}$, 从而破坏细菌的细 胞膜和 DNA, 使细菌死亡, 这可能是纳米复合膜抗 菌性优异的主要原因之一。

\section{4 光催化性能}

甲基橙(MO)是一种有机污染物, 也是应用最广 泛的工业染料之一。实验通过在可见光下降解甲基 橙溶液, 研究纳米复合膜的光催化性能, 纯 PES 膜 (M0)作为参考。如图 5 所示, M0 几乎未发生光降 解, 表明 PES 和无纺布均无光催化性能。相比之下, M1、M2、M3、M4 的脱色率分别为 $9 \% 、 30 \% 、 54 \%$ 、 $63 \%$ 。
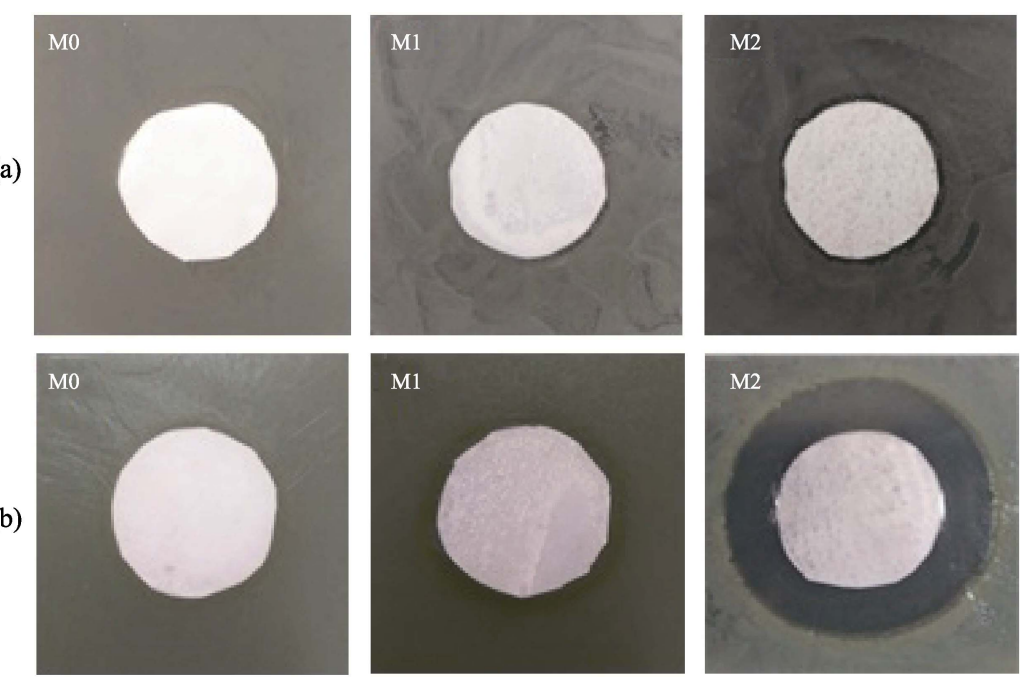
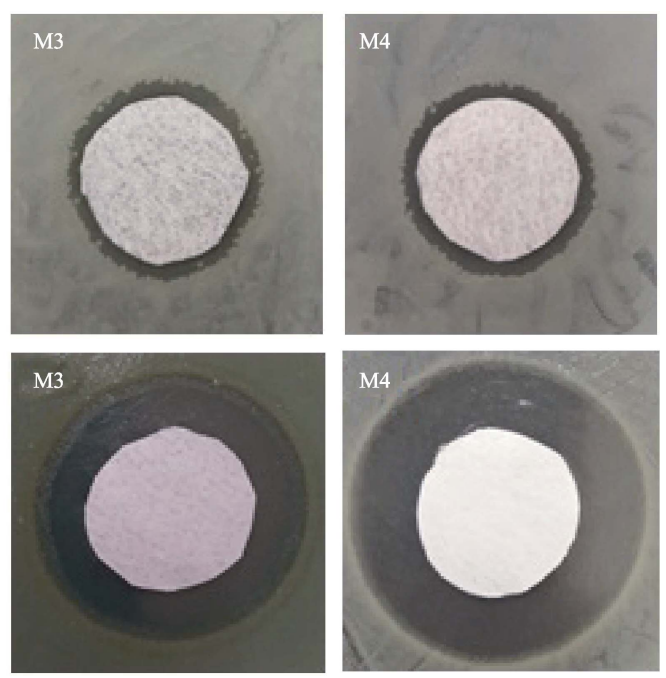

图 4 在抑菌环实验中膜对大肠杆菌(a)和铜绿假单胞菌(b)的抑菌效果

Fig. 4 Antibacterial effect of membranes on (a) E.coli and (b) P. aeruginosa in the disk diffusion test

表 3 纳米复合膜的抗菌性能

Table 3 Antibacterial rate of membranes on E.coli and P. aeruginosa

\begin{tabular}{ccc}
\hline $\begin{array}{c}\text { Membrane } \\
\text { abbreviation }\end{array}$ & PA/\% & EC/\% \\
\hline M0 & 0 & 0 \\
M1 & 61 & 57 \\
M2 & 100 & 76 \\
M3 & 100 & 100 \\
M4 & 100 & 100 \\
\hline
\end{tabular}

图 6 是在可见光照射下, M4 降解甲基橙溶液的 吸光度变化, 可直观地看出, 吸光度变化明显, $464 \mathrm{~nm}$ 处的波峰逐渐下移。这种现象可以间接证明 甲基橙分子被分解成小分子或离子产物。

上述结果皆表明, m-g- $\mathrm{C}_{3} \mathrm{~N}_{4} / \mathrm{Ag}$ 的加入提高了 纳米复合膜在可见光下的光催化活性。复合膜在可 见光下降解甲基橙的机理主要归因于: $\mathrm{m}-\mathrm{g}-\mathrm{C}_{3} \mathrm{~N}_{4} / \mathrm{Ag}$ 产生的光生电子与氧分子反应产生超氧自由基。与 此同时, 由于负载的纳米银的费米能级较低, 易形 


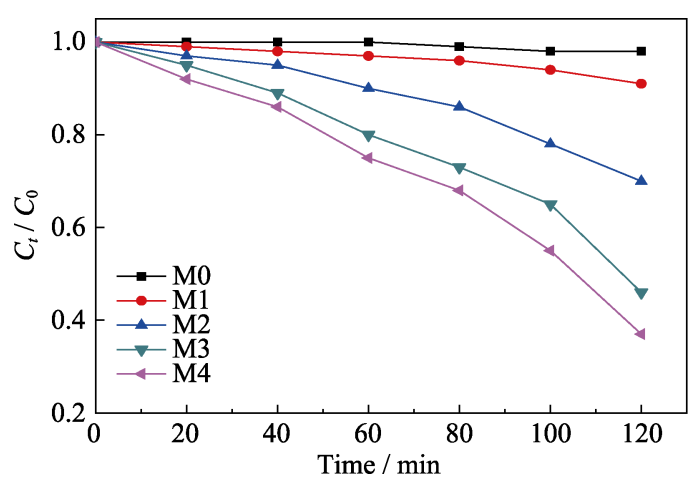

图 5 可见光照射下各种膜降解甲基橙的浓度变化曲线

Fig. 5 Photodegradation of methyl orange with different membranes under visible light irradiation

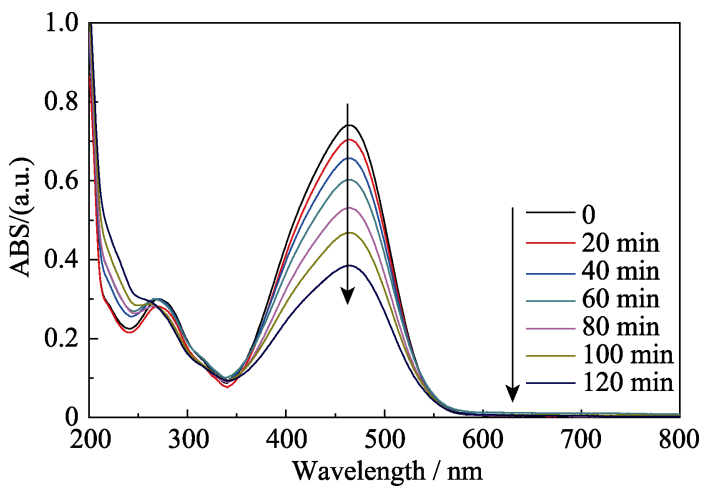

图 $6 \mathrm{M} 4$ 降解甲基橙溶液吸光度变化

Fig. 6 Absorbance variation of methyl orange solution with M4

成肖特基势垒，从而阻止了光生电子与空穴的复合， 提高了复合膜降解甲基橙的效率 ${ }^{[31]}$ 。

\section{5 抗污染性能}

为研究复合膜的抗污染性能, 进行了 BSA 过滤 实验。图 7 展现了膜污染前后、清洗后通过漂洗和 可见光照射后的膜的通量值。可以观察到在 BSA 过 滤期间水通量急剧下降, 这归因于 BSA 在膜表面大 量沉积形成的滤饼层 ${ }^{[32]}$ 。尽管如此, 与 M0 相比纳 米复合膜具有更高的通量, 表明 $\mathrm{m}-\mathrm{g}-\mathrm{C}_{3} \mathrm{~N}_{4} / \mathrm{Ag}$ 的加 入提高了膜的抗污性能 ${ }^{[25]}$ 。经过简单的水洗后, 所

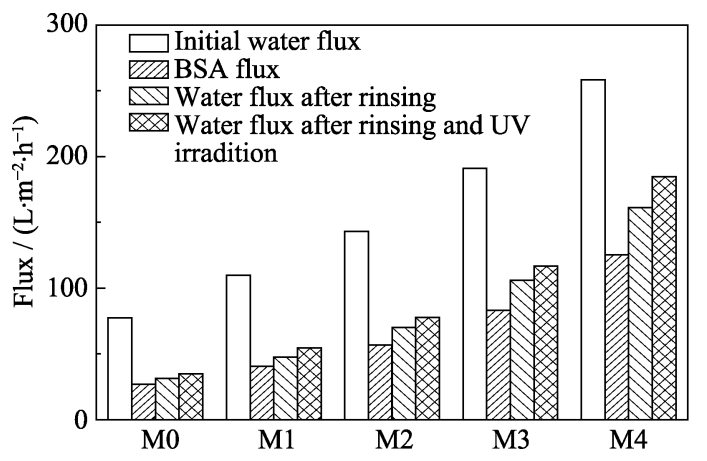

图 7 不同膜在各阶段的通量值

Fig. 7 Flux values of various membranes at different processes
有样品的通量都有不同程度的提高，但通量还远小 于初始纯水通量, 说明水力剪切力可去除部分松散 结合的蛋白质和滤饼层。经水洗和可见光照射后, 复合膜通量进一步增加, 且增长幅度与 $\mathrm{m}-\mathrm{g}-\mathrm{C}_{3} \mathrm{~N}_{4} /$ $\mathrm{Ag}$ 加入量呈正相关性, 表明纳米复合膜在可见光 下的光催化活性和光诱导的亲水性有利于去除强结 合的有机污染物, 并赋予膜自清洁的能力 ${ }^{[33]}$ 。

通量恢复率 $(F R R)$ 是表征膜抗污染性能的重要 参数, 一般认为较高的 $F R R$ 意味着较好的抗污性 能。从图 8 可以看出, 所有复合膜 $F R R$ 皆明显高于 $\mathrm{M} 0$ ，这表明复合膜的抗污染性能随着 $\mathrm{m}-\mathrm{g}-\mathrm{C}_{3} \mathrm{~N}_{4} / \mathrm{Ag}$ 的加入得到改善。经水洗和可见光照射后, 所有膜 的 FRR 皆有所增加, M4 的 FRR 从 $62.36 \%$ 增加至 $71.43 \%$ ，但 M0 的 $F R R$ 增幅不明显，这一现象表明 $\mathrm{m}-\mathrm{g}-\mathrm{C}_{3} \mathrm{~N}_{4} / \mathrm{Ag}$ 和可见光照射能提高膜的抗污性能, 有利于膜的自清洁。

为进一步研究膜污染过程, 利用公式(7) (9)对 总污染率 $\left(R_{\mathrm{t}}\right)$ 、可逆结垢率 $\left(R_{\mathrm{r}}\right)$ 、不可逆结垢率 $\left(R_{\mathrm{ir}}\right)$ 进行计算, $R_{\mathrm{r}}$ 和 $R_{\mathrm{ir}}$ 分别与膜表面松散的蛋白质吸附 和蛋白质在表面的沉积或孔内的截留有关 ${ }^{[34]}$ 。如 图 9 所示, 所有纳米复合膜的 $R_{\mathrm{t}}$ 值明显低于 $\mathrm{M} 0$, 且与 $\mathrm{m}-\mathrm{g}-\mathrm{C}_{3} \mathrm{~N}_{4} / \mathrm{Ag}$ 的加入量呈负相关性, 这是由于 $m-g-C_{3} N_{4} / A g$ 的增加提高了膜表面亲水性。所有样

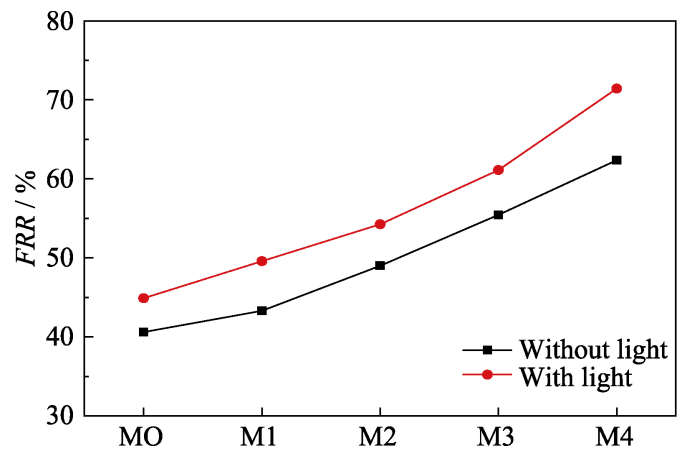

图 8 可见光照射前后的膜通量恢复率 $(F R R)$

Fig. 8 Flux recovery ratio $(F R R)$ of membranes before and after visible light irradiation

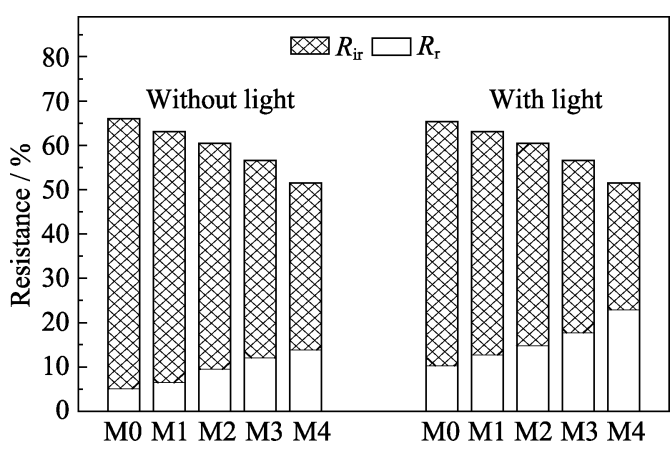

图 9 所有膜的污垢阻力分布

Fig. 9 Distribution of fouling resistance of all membranes 
品在可见光照射后, $R_{\mathrm{ir}}$ 值都有所降低, 这表明 $\mathrm{m}-\mathrm{g}-\mathrm{C}_{3} \mathrm{~N}_{4} / \mathrm{Ag}$ 的加入可以在可见光下对 BSA 进行催 化降解, 有效减轻复合膜的膜污染 ${ }^{[35]}$ 。

\section{3 结论}

1) $\mathrm{m}-\mathrm{g}-\mathrm{C}_{3} \mathrm{~N}_{4} / \mathrm{Ag}$ 的添加改善了纳米复合膜的断 面结构, 并提高了其孔隙率和亲水性。

2) $\mathrm{m}-\mathrm{g}-\mathrm{C}_{3} \mathrm{~N}_{4} / \mathrm{Ag}$ 基纳米复合膜具有优异的抗菌 性能，且对铜绿假单胞菌更具杀菌效果。

3) $\mathrm{m}-\mathrm{g}-\mathrm{C}_{3} \mathrm{~N}_{4} / \mathrm{Ag}$ 的添加显著提高了纳米复合膜 的光催化效果，脱色率由 9\%(M1)提高至 63\%(M4)。

4) $\mathrm{m}-\mathrm{g}-\mathrm{C}_{3} \mathrm{~N}_{4} / \mathrm{Ag}$ 的添加使得纳米复合膜具有光 催化自清洁性能, 并提高了抗污染性能。

\section{参考文献:}

[1] QIAN DONG-LIANG, CHEN DONG-YUN, LI NA-JUN, et al. $\mathrm{TiO}_{2} /$ sulfonated graphene oxide/Ag nanoparticle membrane: in situ separation and photodegradation of oil/water emulsions. Journal of Membrane Science, 2018, 554: 16-25.

[2] GAO JIE, THONG ZHI-WEI, WANG KAI-YU, et al. Fabrication of loose inner-selective polyethersulfone (PES) hollow fibers by one-step spinning process for nanofiltration (NF) of textile dyes. Journal of Membrane Science, 2017, 541: 413-424.

[3] KANG GUO-DONG, GAO CONG-JIE, CHEN WEI-DONG, et al. Study on hypochlorite degradation of aromatic polyamide reverse osmosis membrane. Journal of Membrane Science, 2007, 300(1): $165-171$.

[4] KIM JEONGHWAN, BRUGGEN VAN DER BART. The use of nanoparticles in polymeric and ceramic membrane structures: review of manufacturing procedures and performance improvement for water treatment. Environmental Pollution, 2010, 158(7): 2335-2349.

[5] HUANG JIAN, WANG HUAN-TING, ZHANG KAI-SONG. Modification of PES membrane with $\mathrm{Ag}-\mathrm{SiO}_{2}$ : reduction of biofouling and improvement of filtration performance. Desalination, 2014, 336(1): 8-17.

[6] SHI HENG, HE YI, PAN YANG, et al. A modified mussel-inspired method to fabricate $\mathrm{TiO}_{2}$ decorated superhydrophilic PVDF membrane for oil/water separation. Journal of Membrane Science, 2016, 506: $60-70$.

[7] LI PING, WU WEN-JIAN, LIU JIN-DUN, et al. Investigating the nanostructures and proton transfer properties of Nafion-GO hybrid membranes. Journal of Membrane Science, 2018, 555: 327-336.

[8] KOSEOGLU-IMER DERYA Y, KOSE BORTE, ALTINBAS MAHMUT, et al. The production of polysulfone (PS) membrane with silver nanoparticles (AgNP): physical properties, filtration performances, and biofouling resistances of membranes. Journal of Membrane Science, 2013, 428(12): 620-628.

[9] WEN JIU-QING, XIE JUN, CHEN XIAO-BO, et al. A review on g- $\mathrm{C}_{3} \mathrm{~N}_{4}$-based photocatalysts. Applied Surface Science, 2016, 391: 72-123.

[10] MUÑOZ-BATISTA MARIO J, FONTELLES-CARCELLER OLGA, FERRER MANUEL, et al. Disinfection capability of
$\mathrm{Ag} / \mathrm{g}-\mathrm{C}_{3} \mathrm{~N}_{4}$ composite photocatalysts under UV and visible light illumination. Applied Catalysis B Environmental, 2016, 183(1): $86-95$.

[11] XUE JIN-JUAN, MA SHUAI-SHUAI, ZHOU YUMING, et al. Facile photochemical synthesis of $\mathrm{Au} / \mathrm{Pt} / \mathrm{g}-\mathrm{C}_{3} \mathrm{~N}_{4}$ with plasmonenhanced photocatalytic activity for antibiotic degradation. ACS Applied Materials \& Interfaces, 2015, 7(18): 9630-9637.

[12] GONG YU-TONG, ZHANG PENG-FEI, XU XUAN, et al. A

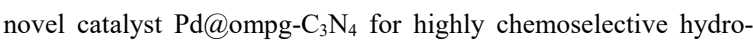
genation of quinoline under mild conditions. Journal of Catalysis, 2013, 297(1): 272-280.

[13] LI YI, LI YA-NAN, MA SHUANG-LONG, et al. Efficient water disinfection with $\mathrm{Ag}_{2} \mathrm{WO}_{4}$-doped mesoporous $\mathrm{g}_{-} \mathrm{C}_{3} \mathrm{~N}_{4}$ under visible light. Journal of Hazardous Materials, 2017, 338: 33-46.

[14] BASRI H, ISMAIL A F, AZIZ M. Polyethersulfone (PES)-silver composite UF membrane: effect of silver loading and PVP molecular weight on membrane morphology and antibacterial activity. Desalination, 2011, 273(1): 72-80.

[15] NGUYEN ANH, ZOU LIN-DA, PRIEST CRAIGN. Evaluating the antifouling effects of silver nanoparticles regenerated by $\mathrm{TiO}_{2}$ on forward osmosis membrane. Journal of Membrane Science, 2014, 454(6): 264-271.

[16] HOSSEIN RAZZAGHI MOHAMMAD, SAFEKORDI ALIAKBAR, TAVAKOLMOGHADAM MARYAM, et al. Morphological and separation performance study of PVDF/CA blend membranes. Journal of Membrane Science, 2014, 470: 547-557.

[17] CAO XUE-LIAN, TANG MING, LIU FEI, et al. Immobilization of silver nanoparticles onto sulfonated polyethersulfone membranes as antibacterial materials. Colloids \& Surfaces B Biointerfaces, 2010, 81(2): 555-562.

[18] ZHU XIAO-YING, TANG LIN, WEE KIN-HO, et al. Immobilization of silver in polypropylene membrane for anti-biofouling performance. Biofouling, 2011, 27(7): 773-786.

[19] IQBAL WAHEED, DONG CHUN-YANG, XING MINGYANG, et al. Eco-friendly one-pot synthesis of well-adorned mesoporous g- $\mathrm{C}_{3} \mathrm{~N}_{4}$ with efficiently enhanced visible light photocatalytic activity. Catalysis Science \& Technology, 2017, 7(8): 1726-1734.

[20] MENG YA-LI, SHEN JU, CHEN DAN, et al. Photodegradation performance of methylene blue aqueous solution on $\mathrm{Ag} / \mathrm{g}-\mathrm{C}_{3} \mathrm{~N}_{4}$ catalyst. Rare Metals, 2011, 30(1): 276-279.

[21] ZHANG MAN-YING, LIU ZI-YA, GAO YONG, et al. Ag modified $g-\mathrm{C}_{3} \mathrm{~N}_{4}$ composite entrapped PES UF membrane with visiblelight-driven photocatalytic antifouling performance. RSC Advances, 2017, 7(68): 42919-42928.

[22] ZHANG MAN-YING, FIELD ROBERT W, ZHANG KAI-SONG. Biogenic silver nanocomposite polyethersulfone UF membranes with antifouling properties. Journal of Membrane Science, 2014, 471(23): 274-284.

[23] LIU SHA-SHA, ZHANG MAN-YING, FANG FANG, et al. Biogenic silver nanocomposite TFC nanofiltration membrane with antifouling properties. Desalination \& Water Treatment, 2016, 57(23): 1-12.

[24] ZHANG MAN-YING, ZHANG KAI-SONG, DE GUSSEME BART, et al. Biogenic silver nanoparticles (bio- $\mathrm{Ag}^{0}$ ) decrease biofouling of bio- $\mathrm{Ag}^{0} / \mathrm{PES}$ nanocomposite membranes. Water Research, 2012, 46(7): 2077-2087.

[25] XU ZHI-WEI, WU TENG-FEI, SHI JIE, et al. Photocatalytic antifouling PVDF ultrafiltration membranes based on synergy of graphene oxide and $\mathrm{TiO}_{2}$ for water treatment. Journal of Mem- 
brane Science, 2016, 520: 281-293.

[26] MÉRICQ J P, MENDRET J, BROSILLON S, et al. High performance PVDF- $\mathrm{TiO}_{2}$ membranes for water treatment. Chemical Engineering Science, 2015, 123: 283-291.

[27] JIN WEN-JIE, HU CHAO-WU, LI LI-HUA, et al, Effect of graphene oxide concentration on morphologies and properties of polysulfone ultrafiltration membranes. Membrane Science and Techology, 2015, 35: 20-24.

[28] LI JIAN-HUA, YAN BANG-FENG, SHAO XI-SHENG, et al. Influence of $\mathrm{Ag} / \mathrm{TiO}_{2}$ nanoparticle on the surface hydrophilicity and visible-light response activity of polyvinylidene fluoride membrane. Applied Surface Science, 2015, 324: 82-89.

[29] ZHANG WEI, ZHOU LI, DENG HUI-PING. Ag modified g- $\mathrm{C}_{3} \mathrm{~N}_{4}$ composites with enhanced visible-light photocatalytic activity for diclofenac degradation. Journal of Molecular Catalysis A Chemical, 2016, 423: 270-276.

[30] ZODROW KATHERINE, BRUNET LENA, MAHENDRA SHAILY, et al. Polysulfone ultrafiltration membranes impregnated with silver nanoparticles show improved biofouling resistance and virus removal. Water Research, 2009, 43(3): 715-723.

[31] ZHAO HUAN-XIN, CHEN SHUO, QUAN XIE, et al. Integration of microfiltration and visible-light-driven photocatalysis on $\mathrm{g}-\mathrm{C}_{3} \mathrm{~N}_{4}$ nanosheet/reduced graphene oxide membrane for enhanced water treatment. Applied Catalysis B Environmental, 2016, 194: 134-140.

[32] MOGHADAM MARYAM TAVAKOL, LESAGE GEOFFROY, MOHAMMADI TORAJ, et al. Improved antifouling properties of $\mathrm{TiO}_{2} / \mathrm{PVDF}$ nanocomposite membranes in UV-coupled ultrafiltration. Journal of Applied Polymer Science, 2015, 132(21): 4173141744.

[33] DAMODAR RAHUL A, YOU SHENG-JIE, CHOU HUI-HSIANG. Study the self cleaning, antibacterial and photocatalytic properties of $\mathrm{TiO}_{2}$ entrapped PVDF membranes. Journal of Hazardous Materials, 2009, 172(2/3): 1321-1328.

[34] XU ZHI-WEI, ZHANG JI-GUO, SHAN MING-JING, et al. Organosilane-functionalized graphene oxide for enhanced antifouling and mechanical properties of polyvinylidene fluoride ultrafiltration membranes. Journal of Membrane Science, 2014, 458(10): $1-13$.

[35] LIN BO, XUE CHAO, YAN XIAO-QING, et al. Facile fabrication of novel $\mathrm{SiO}_{2} / \mathrm{g}-\mathrm{C}_{3} \mathrm{~N}_{4}$ core-shell nanosphere photocatalysts with enhanced visible light activity. Applied Surface Science, 2015, 357: 346-355. 